\title{
Dust Emission in Early-Type Galaxies with the Herschel Virgo Cluster Survey
}

\author{
Sperello di Serego Alighieri ${ }^{1}$ and members of the HeViCS team \\ ${ }^{1}$ INAF - Osservatorio Astrofisico di Arcetri, \\ Largo E. Fermi 5, 50125 Firenze, Italy \\ email: sperello@arcetri.astro.it
}

\begin{abstract}
We have searched for dust in an optical sample of 910 Early-Type Galaxies (ETGs) in the Virgo cluster (447 of which are optically complete at $m_{p g} \leqslant 18.0$ ), extending also to the dwarf ETGs, using Herschel images at 100, 160, 250, 350 and $500 \mu \mathrm{m}$. Dust was found in 52 ETGs (46 are in the optically complete sample), including M87 and another 3 ETGs with strong synchrotron emisssion. Dust is detected in 17\% of ellipticals, $41 \%$ of lenticulars, and in about $4 \%$ of dwarf ETGs. The dust-to-stars mass ratio increases with decreasing optical luminosity, and for some dwarf ETGs reaches values similar to those of the dusty late-type galaxies. Slowly rotating ETGs are more likely to contain dust than fast rotating ones. Only 8 ETGs have both dust and HI, while 39 have only dust and 8 have only HI, surprisingly showing that only rarely dust and HI survive together. ETGs with dust appear to be concentrated in the densest regions of the cluster, while those with HI tend to be at the periphery. ETGs with an X-ray active SMBH are more likely to have dust and vice versa the dusty ETGs are more likely to have an active SMBH.
\end{abstract}

Keywords. galaxies: ISM; galaxies: elliptical and lenticular, cD; ISM: dust, extinction

\section{Introduction}

Most of the baryons in a cluster of galaxies are in the hot intracluster medium, some of which is associated with the most massive galaxies. The hot gas interacts in many ways with the cold phases of the interstellar medium of these galaxies, and these interactions have a fundamental effect on the evolution of the galaxies themselves. In order to understand the evolution of massive galaxies (the topic of this Symposium), particularly in clusters, it is therefore important to study also the coldest phases of their interstellar medium. The most massive galaxies are Early-Type Galaxies (ETGs), and, since they may have formed by merging or accretion of smaller ones, it is useful to include in the study also the dwarf ETGs. In di Serego Alighieri et al. (2007) we have systematically studied the neutral atomic gas (HI) content of a large and complete sample of ETGs in the Virgo cluster, using the Arecibo Legacy Fast ALFA 21-cm survey (Giovanelli et al. 2007). HI is found in very few massive ETGs, where the cold gas could have a recent external origin, and in a few peculiar dwarf galaxies at the edge of the ETG classification. The Herschel Space Observatory (Pilbratt et al. 2010) is giving us the opportunity to study the dust content of the same sample of Virgo ETGs. We have done so within the Herschel Virgo Cluster Survey (HeViCS, Davies et al. 2010), an open-time Key Programme for a confusion-limited imaging survey of a large fraction of the Virgo cluster in 5 bands: at 250, 350 and $500 \mu \mathrm{m}$ with SPIRE (Griffin et al. 2010) and at 100 and $160 \mu \mathrm{m}$ with PACS (Poglitsch et al. 2010). We describe here the main results of this work. A more detailed and complete account has been submitted to A\&A (hereafter dSA12). 


\section{Input sample, analysis and results}

We start with a sample of Virgo ETGs selected in the optical from the GOLDMine compilation (Gavazzi et al. 2003), mostly based on the Virgo Cluster Catalogue (VCC, Binggeli et al. 1985), to be ETG (i.e. equal to or earlier than S0a) and excluding those with $v_{\text {hel }}<3000 \mathrm{~km} / \mathrm{s}$. With these selection criteria 925 ETGs are within the $4 \mathrm{HeViCS}$ fields and constitute our input sample. Out of these, 447 are brighter than the VCC completeness limit $\left(m_{p g} \leqslant 18.0\right)$ and form the optically complete part of our input sample. Out of the input sample, 287 ETGs have inaccurate positions in the literature, based only on the original work of Binggeli et al. (1985), insufficient to find reliable counterparts in the HeViCS images. Using r-band SDSS images, we then remeasured the position for these ETGs, except for 15 (all with $m_{p g}>19.0$ ), for which the identification is unsure. We have looked for a reliable far-IR counterpart in the HeViCS $250 \mu$ m mosaic image for all the 910 Virgo ETGs with accurate coordinates, and found one for 52 of them at $S / N>6$. For these sources we measured the flux in each of the $5 \mathrm{HeViCS}$ bands using an aperture of 30 arcsec radius, large enough to contain the PSF also at 500 $\mu \mathrm{m}$. For 12 sources, which have far-IR emission exceeding this aperture, we used larger apertures, up to 78 arcsec radius. 46 far-IR counterparts have $F_{250} \geqslant 25.4 \mathrm{mJy}$, which is our completeness limit at $250 \mu \mathrm{m}$. We detect dust above the synchrotron component in the 4 ETGs with radio emission, including M87. Given the large number of background sources present in the $250 \mu \mathrm{m}$ images, following the methods of Smith et al. (2011), we estimate that on average 1.5 ETGs (most likely dwarfs), out of our input sample of 910, have a far-IR counterpart which is a background source. We have used the distance given in GOLDMine, which distinguishes various components in the Virgo cluster at 17, 23 and $32 \mathrm{Mpc}$ (Gavazzi et al. 1999).

Dust appears to be very concentrated, much more than stars. The only ETG with a considerable amout of off-nuclear dust is M86, where it appears to be mostly in a filament at 2 arcmin (about $10 \mathrm{kpc}$ in projection) to the South-East (Gomez et al. 2010). Dust masses and temperatures have been estimated for the 52 ETGs with a far-IR counterpart by fitting a modified black-body to the measured far-IR fluxes, assuming a spectral index $\beta=2$ and a MW emissivity, and taking into account colour and aperture corrections. We have also estimated stellar masses with the methods of Zibetti et al. (2009), using the available optical/IR broad-band photometry. The dust temperature ranges between 15 and $30 \mathrm{~K}$, and correlates with the stellar mass and with the B-band average surface brigthness within the effective radius (dSA12). The latter correlation suggests that most of the dust heating is due to radiation produced by stellar sources.

\section{Discussion}

Dust detection rates for the complete samples (i.e. 43 far-IR counterparts with $F_{250} \geqslant$ 25.4 mJy out of the 447 input ETGs with $m_{p g} \leqslant 18.0$ and accurate position) are $9.6 \%$ for all ETGs, $17.1 \%$ for ellipticals, $41.4 \%$ for lenticulars and $3.7 \%$ for dwarf ETGs. The latter rate becomes $3.6 \%$, if we take into account that 1.5 of the assumed far-IR counterpart of dwarf ETGs are in fact counterparts of background sources (see the previous section), and that about 8 of the dwarf ETGs of the input sample without a measured radial velocity are likely background galaxies. These rates are smaller than those previously measured on samples of bright ETGs (Knapp et al. 1989, Temi et al. 2004, Smith et al. 2012), as can be expected since our sample extends to faint galaxies and the dust detection rate correlates strongly with optical luminosity (dSA12). The dust-to-star mass ratio varies over almost 6 orders of magnitude, anticorrelates with the optical luminosity, and for 


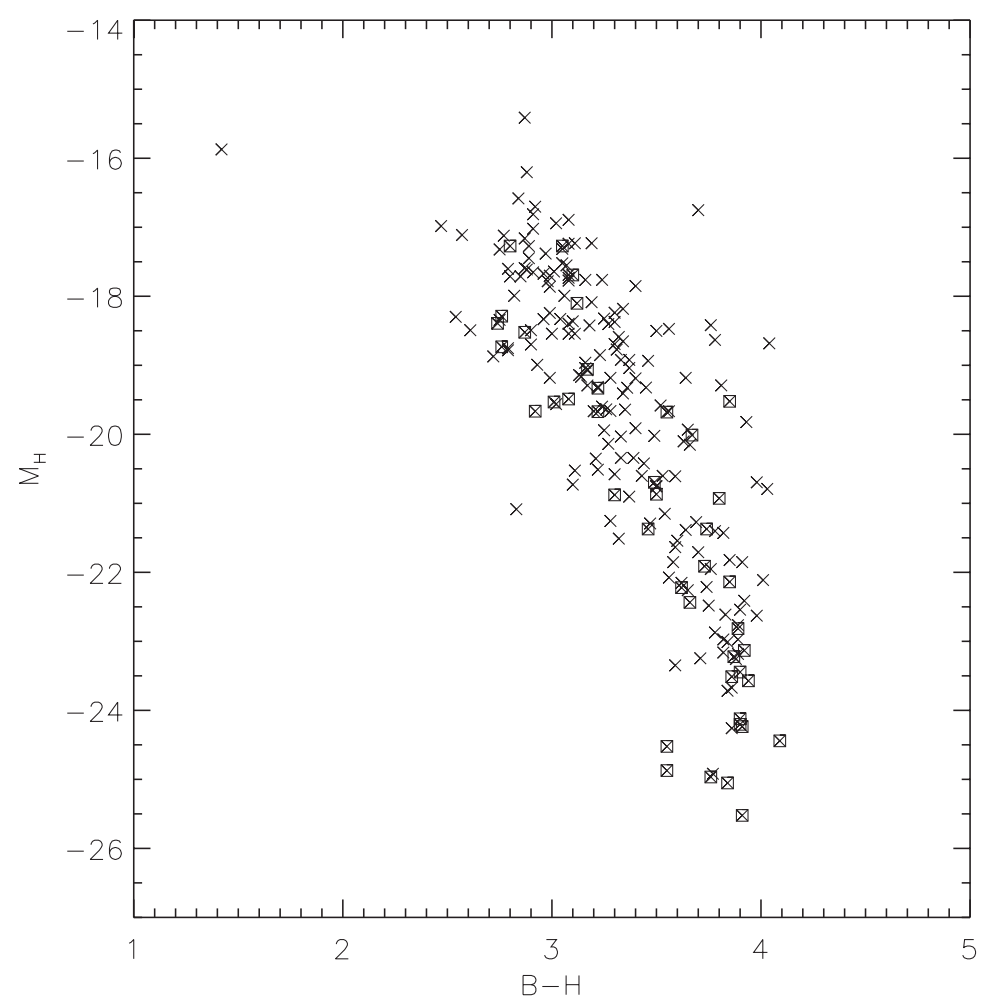

Figure 1. Optical/IR CMD for the Virgo ETGs with accurate photometry. The dusty ones (squares) are not bluer, i.e. not more star-forming, than the other galaxies (crosses).

some dwarf ETGs reaches very high values (around a few $10^{-2}$ ), as high as for the dusty late-type galaxies. This is surprising, also given that the dusty ETGs do not show signs of star formation. In fact the colours of the dusty ETGs are not bluer than those of the non-dusty ones (Fig. 1).

The distinction between fast and slow rotators appears to be an important one for ETGs (Emsellem et al. 2011, and references on the ATLAS ${ }^{3 D}$ project). For the ETGs of our input sample the detailed kinematical information necessary for this distinction is available only for the 49 ones, which are in common with the ATLAS ${ }^{3 D}$ sample. Since we detect dust in $69 \pm 23 \%$ of the slow rotators (in 9 out of 13 ) and in $28 \pm 9 \%$ of the fast ones (in 10 out of 36 ), it appears that the former are considerably more likely to have dust. This is the opposite to what is seen for molecular gas in the whole of the 260 ETGs of the ATLAS $^{3 D}$ sample. In fact Young et al. (2011) find that the CO detection rate is $6 \pm 4 \%$ in slow rotators and $24 \pm 3 \%$ in fast ones. This is surprising, since dust and molecular gas are thought to be closely associated (Draine et al. 2007, Corbelli et al. 2012); in fact, for the dust-detected ETGs of our sample which have information on the molecular gas content, the dust-to-molecular-gas mass ratio is always $2 \times 10^{-2}$ within a factor of two, and lower limits are consistent with this range. We suggest that a possible explanation of this difference could be an environmental effect, since most of the ATLAS ${ }^{3 D}$ galaxies are outside of the Virgo cluster. In fact, of the 19 dust-detected ETGs, which we have in common with the ATLAS ${ }^{3 D}$ sample, molecular gas is detected in 3 slow rotators and in 5 fast ones, a much more balanced situation than found by Young et al. (2011) in the whole $\operatorname{ATLAS}^{3 D}$ sample, and all slow rotators with molecular gas in this whole sample are actually in the Virgo cluster. The difference we find could be due to the presence of 
kinematically peculiar objects among the dusty slow rotators in the Virgo cluster, like galaxies with counter-rotating components mimicking slow rotation. We can exclude this possibility, since the brightest and most regular ellipticals and lenticulars in the Virgo cluster like M49, M84, M86, M87, M89, NGC 4261 and NGC 4526 are among the dusty slow rotators, reinforcing our suggestion about an environmental effect.

We have also looked at the relationship between dust and HI for the Virgo ETGs, updating the work done by di Serego Alighieri et al. (2007) on the HI content of Virgo ETGs to include the 4-8 degrees declination strip, which has become available in the ALFALFA HI survey since their work (Haynes et al. 2011). We find an intriguing incompatibility between dust and HI in Virgo ETGs: we detect both dust and HI in only 8 ETGs, while 39 ETGs have dust but no HI, and 8 have HI but no dust. This dichotomy between dust and $\mathrm{HI}$ is reinforced by the position of the parent galaxies in the cluster. Dusty ETGs appear to concentrate in the densest regions of the Virgo cluster, while HI-rich ETGs tend to be at the periphery. While the presence of ETGs with dust but no HI can be explained by the longer dust survival times and by the stronger effects of ram pressure stripping on HI, more difficult to understand is that there are ETGs with HI but no dust, also given that these include two rather bright S0 galaxies: NGC 4262 and NGC 4270.

Concerning the relationship between the presence of dust and that of an AGN in Virgo ETGs, our input sample has 71 ETGs in common with the sample of Virgo ETGs for which Gallo et al. (2010) have looked for the presence of a supermassive black-hole (SMBH) by observing with Chandra the nuclear X-ray luminosity down to a few $10^{38} \mathrm{erg} / \mathrm{s}$. Out of the 71 common ETGs, 25 (35\%) have X-rays, most likely from a $\mathrm{SMBH}$, and 14 (20\%) have dust. Of the X-ray luminous ETGs 36\% have dust, and out of the dusty ETGs $64 \%$ are X-ray luminous. It appears that ETGs with an X-ray active $\mathrm{SMBH}$ are more likely to have dust, and that ETGs with dust are more likely to have an X-ray active SMBH.

\section{References}

Binggeli, B., Sandage, A., \& Tammann, G. A., 1985, AJ, 90, 1681

Corbelli, E., Bianchi, S., Cortese, L., et al., 2012, A\&SA, 542, A32

Davies, J. I., Baes, M., Bendo, G. J., et al., 2010, $A \& A$, 518, L48

di Serego Alighieri, S., Gavazzi, G., Giovanardi, C., et al., 2007, A\&3A, 474, 851

Draine, B. T., Dale, D. A., Bendo, G., et al., 2007, ApJ, 663, 866

Emsellem, E., Cappellari, M., Krajnović, D., et al., 2011, MNRAS, 414, 888

Gallo, E., Treu, T., Marshall, P. J., et al., 2010, ApJ, 714, 25

Gavazzi, G., Boselli, A., Scodeggio, M., Pierini, D., \& Belsole, E., 1999, MNRAS, 304, 595

Gavazzi, G., Boselli, A., Donati, A., Franzetti, P., \& Scodeggio, M., 2003, A\&A, 400, 451

Giovanelli, R., Haynes, M. P., Kent, B. R., et al., 2007, AJ, 133, 2569

Gomez, H. L., Baes, M., Cortese, L., et al., 2010, A\&SA, 518, L45

Haynes, M. P., Giovanelli, R., Martin, A. M., et al., 2011, AJ, 142, 170

Knapp, G. R., Guhathakurta, P., Kim, D.-W., \& Jura, M., 1989, ApJSS, 70, 329

Pilbratt, G. L., Riedinger, J. R., Passvogel, T., et al., 2010, A\&AA, 518, L1

Smith, D. J. B., Dunne, L., Maddox, S. J., et al., 2011, MNRAS, 416, 857

Smith, M. W. L., Gomez, H. L., Eales, S. A., et al., 2012, ApJ, 748, 123

Temi, P., Brighenti, F., Mathews, W. G., \& Bregman, J. D., 2004, ApJSS, 151, 237

Young, L. M., Bureau, M., Davis, T. A., et al., 2011, MNRAS, 414, 940

Zibetti, S., Charlot, S., \& Rix, H.-W., 2009, MNRAS, 400, 181 